\title{
Aproximación preliminar a la simulación de la dispersión sonora en asentamientos arqueológicos
}

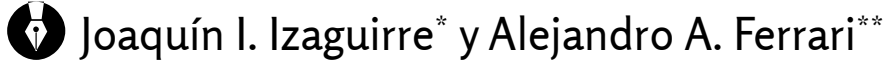

Recibido: 10 de julio de 2017

Aceptado: 26 de febrero de 2018

\section{Resumen}

Las aproximaciones arqueológicas a los paisajes y lugares se han concentrado mayoritariamente en la permeabilidad visual de los espacios, postergando otras modalidades sensoriales. Esta tendencia se ha revertido gradualmente con aportes de diversas disciplinas como la geografía, la proxémica, la sociología, la ingeniería acústica y la arqueología. En este trabajo presentamos el modo en que buscamos combinar estudios tradicionales de visibilidad con simulaciones acústicas realizadas partiendo de modelos tridimensionales. A partir de un caso de estudio sobre el sitio Las Pailas (valle Calchaquí norte, Provincia de Salta, Argentina), exploramos dos escenarios hipotéticos que combinan análisis de visibilidad con simulaciones acústicas. Los resultados, aunque preliminares, indican que la incorporación de análisis de dispersión sonora puede resultar útil a la hora evaluar la permeabilidad de recintos domésticos y espacios públicos. Además, nos permitieron identificar algunas limitaciones y señalar futuras direcciones.

\section{A preliminary approach to sound propagation simulation in archaeological settlements}

\footnotetext{
Abstract

Archaeological approaches to landscapes and places have traditionally focused on the visual permeability of enclosures, leaving aside other sensory modalities. This dominant trend has been gradually challenged by contributions from various disciplines such as geography, proxemics, sociology, acoustic engineering and archeology itself. In this paper, we present the way in which we sought to combine traditional visibility analysis with acoustic simulations using 3D-models. Based on a case study from the archaeological settlement of Las Pailas (North Calchaquí Valley, Salta province, Argentina), we explored two hypothetical settings that combine visibility analysis with acoustic simulations. Results, though preliminary, indicate that including sound

Keywords

Senses 3D-modeling Multi-physical simulations Architecture

\section{Palabras clave}

Sentidos

Modelado tridimensional Simulaciones multifísicas Arquitectura 
dispersion analysis might be useful in further assessing the permeability of public and domestic enclosures. It also allowed us to identify some limitations and future research directions.

\section{Introducción}

A partir del giro espacial que han dado los estudios críticos en ciencias sociales, el desarrollo de proyectos como el World Soundscape Project (ver Schafer, 1969), los enfoques postmodernos en arqueología, la incorporación de la proxémica (Hall, 1959) y la determinación de isovistas (sensu Benedikt, 1979) como metodologías de análisis espacial, se han consolidado aquellas investigaciones arqueológicas que buscan comprender el modo en que paisajes y lugares se encuentran configurados de modo que promueven o inhiben diferentes modalidades sensoriales. De esta forma, se ha virado hacia una conceptualización del espacio vivido que enfatiza el modo en que paisajes y lugares intersectan y transforman las biografías experienciales de los sujetos y las sociedades.

En esta línea de investigación se ha planteado la necesidad de una arqueología de los sentidos, comprendida como un intento

(...) por entender cómo las personas producen sus subjetividades, sus identidades fundadas colectiva y experiencialmente, cómo viven sus rutinas diarias y construyen sus propias historias a través de la experiencia sensorial de la materia, de otros seres animados e inanimados, humanos, animales, plantas $u$ otros (...) (Hamilakis, 2011, p. 208, traducción de los autores).

La arqueología de los sentidos es, en definitiva, un modo más de acercarse al modo en que las personas reproducían sus subjetividades e identidades colectivas a partir del estudio de su imbricación en el mundo material y espacial.

Partimos de que la percepción es la conjunción entre un proceso cultural y fenómenos físicos y fisiológicos. Siguiendo a Classen (1997) se propone que "ver, oír, tocar, degustar y oler no sólo son medios de aprehensión de los fenómenos físicos sino además avenidas para la trasmisión de los valores culturales" (Classen, 1997, p. 41). Por lo tanto, la aprehensión del mundo va a cambiar en función del valor que cada sociedad le asigne a cada una de las modalidades sensoriales, exaltando la importancia cultural y simbólica de ciertos sentidos sobre otros (ver por ejemplo Seeger, 1975).

Diversos investigadores han buscado complementar los análisis visuales integrando otros sentidos (ver por ejemplo Sanders, 1990 para olfato; Feld, 1996; Ferrari, Leibowicz, Izaguirre y Acuto, 2017; Kolar, Rick, Cook y Abel, 2012; Scullin y Boyd, 2014 para sonido; Nyberg, 2010 para tacto, entre muchos otros ejemplos). Se ha señalado que enfocarse en una única modalidad sensorial ignora elementos que pudieron ser claves a la hora de producir y reproducir elementos esenciales de la experiencia humana (Cripps, 2007; Van Ede, 2009), por lo que es necesario explorar aproximaciones metodológicas que trasciendan el sentido de la vista.

Con el advenimiento y la creciente accesibilidad de tecnologías y programas informáticos destinados al modelado tridimensional y multifísico, es posible acercarnos a la superación de aquella falencia incorporando otras modalidades sensoriales y examinando su co-ocurrencia. El objetivo principal de este trabajo es exponer el potencial de esta clase de aproximaciones para determinar las propiedades de los ambientes construidos. Ejemplificaremos esta exploración preliminar con un área del sitio Las Pailas (SalCac 18) en el valle Calchaquí norte (Salta, Argentina). En este caso integraremos el modelado tridimensional del terreno y de la arquitectura de un 
espacio público y de los módulos residenciales aledaños, la proyección de isovistas, renderizados, y el modelado de la dispersión de sonido.

Expondremos aquí los materiales utilizados, las variables consideradas en el modelado y los resultados preliminares obtenidos a partir de evaluar dos escenarios de simulación. Mostraremos el modo en que su aplicación a la arqueología puede enriquecer nuestro entendimiento respecto de la incidencia de la arquitectura en la promoción e inhibición de ciertos sentidos.

\section{El caso de estudio: el sitio arqueológico Las Pailas (SSalCac 18)}

Las Pailas es un poblado conglomerado cuya ocupación se remonta mayoritariamente al período Tardío (1000-1450 d.C.). Las primeras menciones que hallamos sobre el sitio se remontan a las exploraciones realizadas por Pío Pablo Díaz quien, en 1964, fue informado de la existencia del lugar por parte de pobladores locales (Tarragó, 1977). Fue oficialmente reconocido como sitio en el año 1969, y el área que fuera considerada como residencial fue registrada como SSalCac 18 (Tarragó, 1977). Las primeras excavaciones estuvieron a cargo del equipo de Víctor Núñez Regueiro y Myriam Tarragó a través de dos campañas realizadas mediante subsidios provinciales del Gobierno de Salta (Tarragó y Núñez Regueiro, 1972). Durante el 2012 se realizaron nuevos sondeos, excavaciones y la planimetría de gran parte del área de residencias (Figura $1^{1}$ ). Esta área se compone fundamentalmente de módulos de estructuras articuladas concentradas en sectores que están delimitados por vías de circulación (ver Kergaravat, Ferrari y Acuto, 2015). Se destacan además seis posibles espacios públicos de magnitud dentro del sitio.

Nuestro interés en esta oportunidad no está en recrear las experiencias que pudieron haber tenido las personas en el pasado sino en evaluar la forma en la que la arquitectura pudo afectar la dispersión sonora y la proyección de la vista. Con este fin, construimos un modelo tridimensional de un sector del sitio y evaluamos dos escenarios.

En el primer escenario se busca comprender las posibilidades para la dispersión sonora en y desde el Espacio Público 1 (EP1 de aquí en adelante, Figura 1) y la proyección de la vista cuando se ingresa en él. Este espacio fue escogido porque posee excavaciones previas (nos permite corroborar que la pendiente de la superficie actual guarda cierta relación con la pendiente que pudo haber tenido originalmente) y porque se encuentra flanqueado por un sendero sobreelevado hacia el lado norte y un amplio conjunto de estructuras en el lado sur (no intercaladas por un sendero). Esto nos permitió concentrarnos en un único espacio a la vez que evaluar dispersiones distintivas hacia el exterior. Dentro de este escenario tenemos interés en analizar: 1) el alcance del sonido en el interior de la plaza cuando es emitido desde el extremo noroccidental y desde el suroriental a fin de identificar si existen diferencias en la dispersión sonora en función de la localización de la fuente emisora; 2) la forma en la que los accesos podrían haber permitido o inhibido la vista y el sonido cuando sujetos insertos al modelo ingresan en el espacio público y 3 ) evaluar el alcance de los sonidos emitidos desde el espacio público, estimando en qué recintos aledaños se hubiese escuchado.

En este escenario, para analizar las vistas se realizaron análisis de cuencas visuales y renderizados tanto desde los accesos hacia el interior del EP1 como desde los emisores de sonido. Para ello construimos un modelo tridimensional en el que además se situaron dos emisores (uno en cada extremo longitudinal de la plaza) que emiten 4.000.000 de vectores acústicos cada uno. Adicionalmente, se integraron al modelo 51 sondas cilíndricas de $80 \mathrm{~cm}$ de alto y $40 \mathrm{~cm}$ de diámetro elevadas $80 \mathrm{~cm}$ respecto
1. Consideramos que es un plano parcial debido a que se encuentra restringido a lo que consideramos como núcleo del poblado. El sitio en su totalidad, incluyendo los campos de cultivo, tiene una extensión estimada en 300 ha (Tarragó, 1977) y podría tener más aún. 


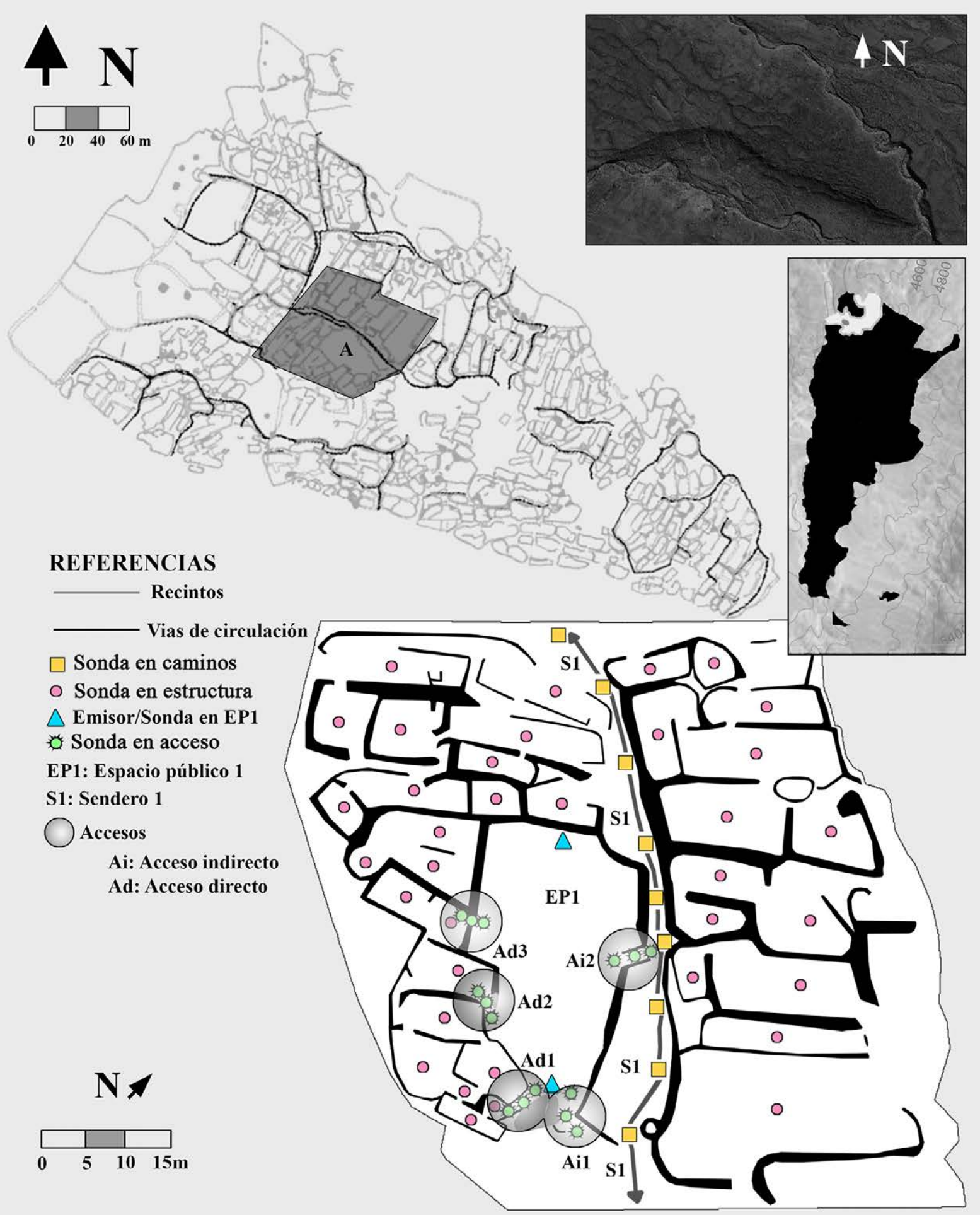

Figura 1. Localización de Las Pailas, plano parcial del sitio, vías de circulación, accesos y sector analizado durante este trabajo indicando la distribución de las sondas utilizadas en los dos escenarios. 
de la superficie. Éstas registran el nivel de presión sonora en el punto en que se encuentran. Quince sondas se instalaron en los cinco accesos y 36 sondas en cada uno de los recintos que se encuentran alrededor del espacio público (Figura 1).

El segundo escenario analiza lo que podrían haber visto y oído los sujetos que circulaban por el sendero 1. Para el análisis visual se realizó un estudio de visibilidad acumulada a lo largo de su recorrido. Para la dispersión sonora, se situaron 13 emisores de sonido en las estructuras que bordean el camino y se agregaron nueve sondas a través del sendero (Figura 1).

\section{Construcción del modelo para la evaluación de los escenarios propuestos}

El principal problema que enfrentamos derivó del emplazamiento de Las Pailas. Sus recintos están construidos sobre una pendiente longitudinal media de $8^{\circ}$ con una diferencia altitudinal de 56,26 m entre el punto de mayor altitud al Noroeste y de menor altitud al Sureste ( $\gamma$ en Figura 2). Además, presenta otras dos pendientes ( $\alpha$ y $\beta$ en Figura 2) con sentido suroeste-noreste. Estas pendientes generan zonas con conjuntos de estructuras que se disponen en diferentes niveles altitudinales. A grandes rasgos, crea una zona elevada central con dos zonas deprimidas hacia los laterales (ver perfil 2-1 y 3-4 en Figura 2) por lo que existen una serie de consideraciones a realizar para construir los escenarios planteados.

En primer lugar, fue necesario crear un modelo digital de elevación (MDE) que integre los muros como variable altimétrica. A partir de los puntos tomados con estación total se construyó un modelo Triangulated Irregular Network (TIN) (Figura $3 \mathrm{~A})$ que representa de forma aproximada los contornos de las estructuras. La cantidad de excavaciones realizadas no nos permiten definir con precisión la altura de cada recinto presente en el sitio. No obstante, en función de las excavaciones realizadas por Tarragó (1977) y de las realizadas por nosotros, creemos que las alturas de los muros podrían haber oscilado entre 1,4 y 1,6 m para los recintos habitacionales y entre $0,7 \mathrm{~m}$ y $1,2 \mathrm{~m}$ para algunos muros del espacio público 1 . Para correr los análisis requeridos fue necesaria la incorporación de puntos con valores intermedios que permitieran que el mallado del modelo respete los límites precisos de las superficies y la altura de los muros.

Para ello, se ingresaron manualmente los puntos necesarios siguiendo el contorno de los recintos. Se repitió el procedimiento ingresando el contorno interno y externo de los muros en función de la altura estipulada de las paredes quedando estas integradas en el modelo tridimensional.

El nuevo mapa de curvas de nivel con los puntos intermedios (Figura 3B) permitió crear un MDE que contempla la variación altitudinal cada $5 \mathrm{~cm}$ (Figura 3C). Este plano fue utilizado para correr los análisis de visibilidad y se utilizó para generar un TIN de mayor resolución (Figura 3D) sobre el cual se modeló la dispersión sonora. Para las reconstrucciones tridimensionales y renderizados se adaptó este modelo reemplazando los muros geométricos por muros simulados de rocas y se le agregaron texturas similares a las rocas presentes en los muros del sitio (Figura 3E). Para cada análisis particular se utilizaron además diversos programas. Los renderizados fueron realizados en Autodesk $3 d$ Max 2017, las simulaciones de la dispersión sonora fueron realizadas en Comsol Multiphysics 5.2 y los análisis de visibilidad acumulada y de cuencas visuales fueron generadas en ESRI ArcMap 10.2 y en Autodesk Civil 3D. El equipamiento utilizado fue un ordenador con procesador Intel I7 4770K, $32 \mathrm{~Gb}$ de memoria RAM y una placa de video dedicada con 3 Gb DDR 5 y 3,85 teraflops. 


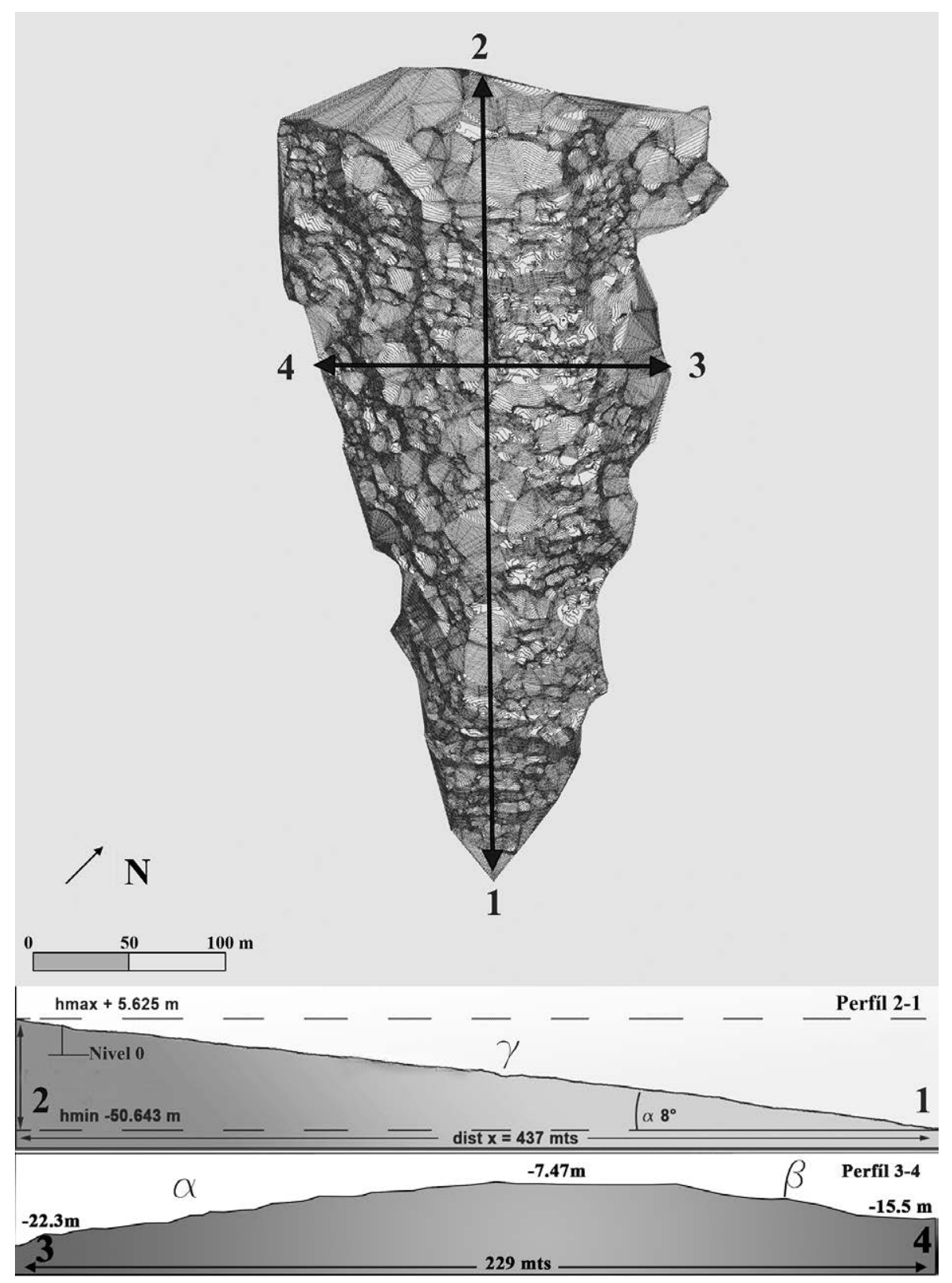

Figura 2. Curvas de nivel $(0,1 \mathrm{~m})$ de Las Pailas y perfiles de las pendientes generales del sitio.

\section{Variables fundamentales comunes a los escenarios}

Las variables ambientales consideradas para las simulaciones acústicas son la temperatura y la presión atmosférica. Para la primera utilizamos un valor promedio de $20^{\circ}$ C. La segunda fue estipulada en función de la altitud del sitio (3.200 m s.n.m.) y se estableció en 0,672 atmósferas.

La principal variable física empleada en las simulaciones acústicas es la velocidad del sonido en los diferentes materiales. Para el aire se utilizó el valor de $344 \mathrm{~m} / \mathrm{s}$ (Forniash,

2. La velocidad del sonido en el granito varía en función de su composición mineralógica, por dicha razón se utiliza el valor máximo de $6.000 \mathrm{~m} / \mathrm{s}$. 2015). Para el caso de las rocas se empleó el valor máximo de las rocas graníticas $\left(6.000 \mathrm{~m} / \mathrm{s}^{2}\right.$ en Forinash, 2015), materia prima principal de los muros de Las Pailas. El coeficiente de absorción acústica del suelo fue fijado en 0,04 , mientras que para los muros se utilizó un valor de 0,02 (Cátedra de acondicionamiento acústico, 2010). 

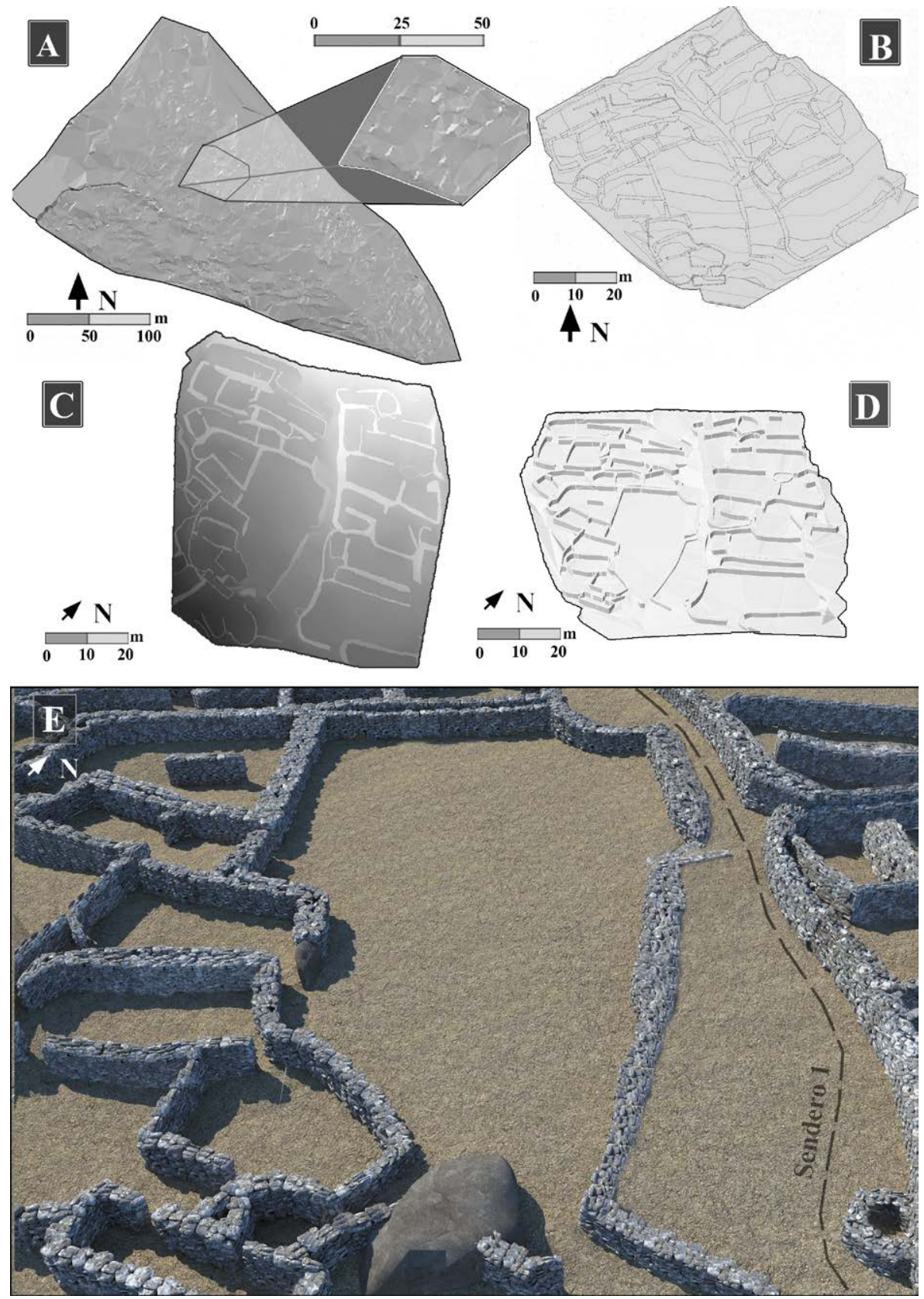

Figura 3. A) Modelo TIN a partir de los puntos originales; B) Nube de puntos resultante de la incorporación de los puntos intermedios y curvas de nivel ajustadas; C) MDE generado a partir de la nueva nube de puntos; D) Modelos TIN realizados a partir de la nueva nube de puntos; E) Modelo 3D texturizado realizado en 3D Max a partir del modelo TIN.

La fuente de sonido simulada es la de la voz humana en $70 \mathrm{~dB}$ a $0,65 \mathrm{~m}$. Para la frecuencia de la voz humana se utilizó el valor promedio de $100 \mathrm{~Hz}$ (Parrondo Gayo, Velarde Suárez, González Pérez, Ballesteros Tajadura y Santolaria Morros, 2006). La dispersión del sonido se produce desde la sonda emisora (Figura 4B) y es registrado ${ }^{3}$ por todas las superficies (Figura 4A).

Para determinar si el sonido es percibido o no por cada una de las sondas instaladas en nuestros escenarios es necesario considerar las curvas isofónicas, ya que nos permiten determinar a qué nivel de presión sonora un sonido es audible en determinada

3. Los decibeles registrados son el resultado de la suma de las reflexiones, refracciones y transmisiones de los vectores acústicos sobre las diferentes superficies y no corresponden solamente aquellos decibeles registrables en una situación hipotética de campo abierto (es decir, sin obstáculos, que a su vez ofician de superficies adicionales de reflexión). 

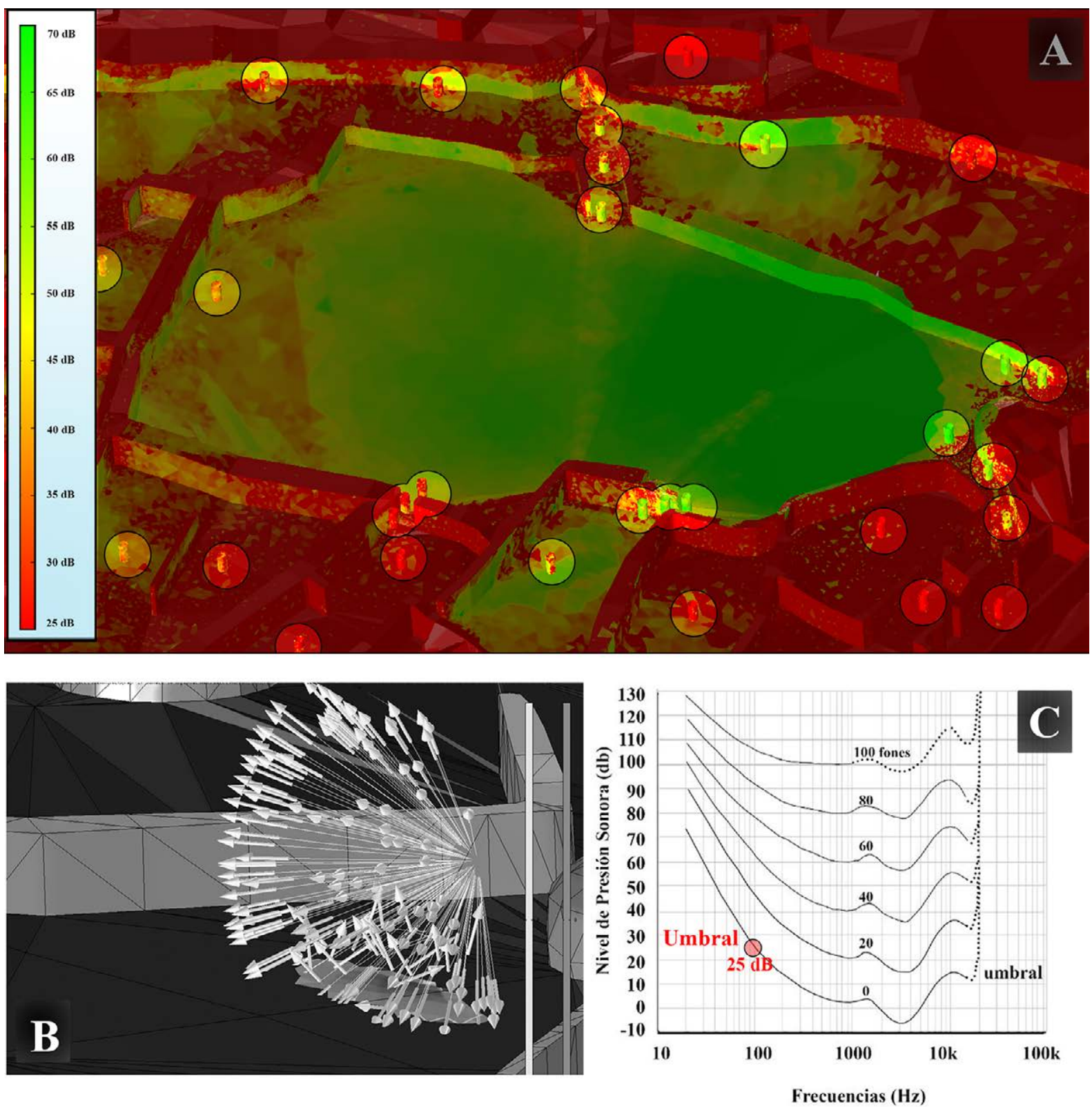

Figura 4. A) Registro del nivel de presión sonora por las diferentes sondas y superficies; $B$ ) Dispersión vectorial desde fuente la emisora; C) Curvas isofónicas para diferentes frecuencias (ISO 226, 2003) y umbral de audibilidad para los $100 \mathrm{~Hz}$.

frecuencia. Se utilizan aquí las curvas ISO 226:2003 (Figura 4C) generadas por The research Institute of Electrical Communication de la Universidad de Tohoku, Japón (Takeshima, Susuki, Ozawa, Kumagai y Sone, 2003). Para la frecuencia de la voz humana que consideramos aquí, $100 \mathrm{~Hz}$, el umbral de audición se encuentra en los $25 \mathrm{~dB}$.

\section{Escenarios analizados}

\section{Escenario 1: Dispersión sonora y proyección visual desde y hacia el EP1}

El espacio público aquí testeado se encuentra ubicado en una posición relativamente central respecto de la distribución arquitectónica del poblado (ver Figura 1). Con 
una superficie de $510 \mathrm{~m}^{2}$ y un perímetro de $106 \mathrm{~m}$, alcanza los $38,12 \mathrm{~m}$ en su máxima longitud y los 21,6 m en su máxima amplitud. Respecto a las alturas de las estructuras que rodean el EP1, actualmente podemos estimar que el nivel de ocupación de EP1 se encontraba un metro por arriba de las estructuras al Sur y un metro por debajo de la vía de circulación al NE. Adicionalmente, EP1 cuenta con cinco accesos: uno de ellos se encuentra relacionado con la vía de circulación al Norte y al Noreste. Otro acceso al Sureste lo vincula con un gran espacio desprovisto de estructuras. Finalmente, tres accesos lo vinculan con una serie de estructuras situadas al Suroeste (ver Figura 1). Su superficie se encuentra en pendiente noroeste-sureste con tres metros de diferencia altitudinal de extremo a extremo y una pendiente de $4^{\circ}$.

Para evaluar este escenario expondremos los resultados de la dispersión sonora al interior del EP1 y su repercusión en los accesos y en las estructuras circundantes. Para ello introdujimos al modelo un emisor en cada extremo del eje longitudinal de la plaza a fin de cubrir la totalidad del espacio. Para cada emisor, elegimos un modo de dispersión cónica en dirección al extremo opuesto del espacio y configuramos una emisión de cuatro millones de vectores acústicos a fin de cubrir la mayor superficie de dispersión posible.

\section{Interior del EP1}

Los emisores 1 y 2 están situados en los extremos longitudinales de la Plaza (Figura 5A). Cuando el sonido es emitido desde el emisor 1, el emisor 2 registra 50,3 dB con una pérdida de 19,7 dB (Figura 5B). Cuando el sonido es emitido desde el emisor 2, el emisor 1 registra 46,9 dB con una pérdida de 23,1 dB (Figura 5C). Podemos observar que ambos registraron lecturas por encima del umbral de audición (25 dB). Para complementar el análisis de dispersión acústica de estos dos emisores se realizaron renderizados desde la posición de cada emisor (Figura 5B y 5C). En este caso encontramos que no existen diferencias sensibles entre lo que los dos emisores podrían ver y escuchar.

\section{Accesos}

Para evaluar los accesos se clasificaron según fueran directos e indirectos. Los primeros incluyen aquellos que comunican el EP1 con otros recintos. Los segundos, en cambio, incluyen aquellos que lo comunican con áreas transitables como ser vías de circulación lindantes (ver Figura 1). Como ya fuera realizado en otra oportunidad (Ferrari et al., 2017), aplicamos un criterio metodológico que se corresponde con diferentes instancias de acercamiento al espacio testeado: aproximación (antes de ingresar al recinto), trascendencia (cuando se está atravesando el acceso) y permanencia (una vez adentro del recinto). Para cada instancia, el modelo tridimensional tiene una sonda de registro de nivel de presión sonora. Para determinar el porcentaje de las superficies visibles del EP1 se corrieron análisis de cuencas visuales desde cada instancia (Tabla 1). Adicionalmente, se realizaron renderizados desde las instancias de aproximación de cada acceso (Figura 6). Los resultados de la simulación de la dispersión sonora se indican en la Figura 6.

\section{Acceso indirecto 1}

Se encuentra ubicado al sureste del EP1 (ver Figura 1). La cuenca visual desde los observadores en estas tres instancias es notoriamente alta, con valores de un $91 \%$ para la instancia de aproximación y 93\% y 97\% para las instancias de trascendencia y permanencia respectivamente. En cuanto al modelo de dispersión sonora, cuando la fuente del sonido es el emisor 1 se obtuvieron valores de 53,3 dB para
4. De acuerdo a estándares psicoacústicos (ver por ejemplo, Scharf, 1978), un cambio de hasta $3 \mathrm{~dB}$ resultaría imperceptible mientras que un incremento de 10 $\mathrm{dB}$ implica el doble del volumen percibido, al tiempo que un cambio de $20 \mathrm{~dB}$ implica el cuádruple. 

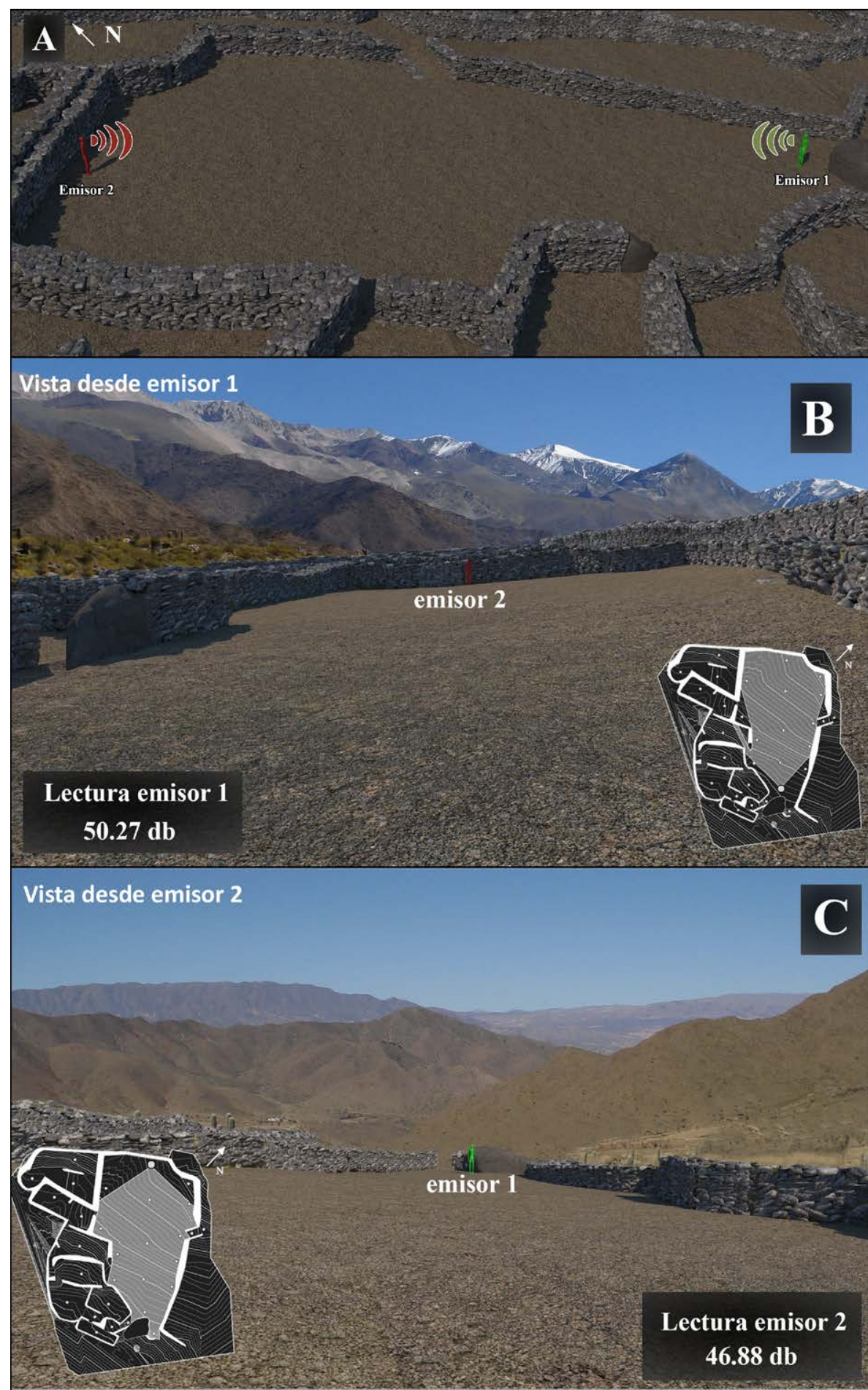

Figura 5. A) Ubicación de las sondas emisoras y valores registrados por cada una de ellas; $B$ y $C$ ) Renderizados y lecturas registradas por el emisor 1 y 2.

la aproximación, $63,8 \mathrm{~dB}$ para la trascendencia y $61,41 \mathrm{~dB}$ para la permanencia. Cuando la fuente del sonido es el emisor 2 los valores para la aproximación fueron de $43 \mathrm{~dB}, 39,75 \mathrm{~dB}$ para la trascendencia y $40 \mathrm{~dB}$ para la permanencia. Es decir, las voces generadas en el EP1 son audibles desde las tres instancias independientemente de la fuente de emisión. 


\begin{tabular}{|l|c|c|c|c|c|c|}
\cline { 2 - 7 } \multicolumn{1}{c|}{} & \multicolumn{2}{c|}{ Aproximación } & \multicolumn{2}{c|}{ Trascendencia } & \multicolumn{2}{c|}{ Permanencia } \\
\hline Accesos & $\begin{array}{c}\text { Superficie visible } \\
\left(\mathrm{m}^{2}\right)\end{array}$ & $\begin{array}{c}\text { Porcentaje de } \\
\text { visibilidad }\end{array}$ & $\begin{array}{c}\text { Superficie visible } \\
\left(\mathrm{m}^{2}\right)\end{array}$ & $\begin{array}{c}\text { Porcentaje de } \\
\text { visibilidad }\end{array}$ & $\begin{array}{c}\text { Superficie visible } \\
\left(\mathrm{m}^{2}\right)\end{array}$ & $\begin{array}{c}\text { Porcentaje de } \\
\text { visibilidad }\end{array}$ \\
\hline Indirecto 1 & 457,59 & 91,4 & 475,76 & 93 & 496,76 & 97 \\
Indirecto 2 & 398,8469 & 78 & 475,25 & 93 & 506,65 & 99 \\
Directo 1 & 22,3257 & 4 & 465,26 & 91 & 480,63 & 94 \\
Directo 2 & 103,88 & 20 & 108,39 & 21 & 412,68 & 81 \\
Directo 3 & 123,31 & 24 & 404,45 & 79 & 428,071 & 84 \\
\hline
\end{tabular}

\begin{tabular}{|l|l|l|}
\hline Superficie EP1 & 510 & $\mathrm{~m}^{2}$ \\
\hline
\end{tabular}

Tabla 1. Visibilidad desde cada observador.

Cuando la fuente de emisión es el emisor 1, la diferencia entre estar tras el acceso indirecto 1 y estar en el espacio público pudo haber sido notable $(+8,11 \mathrm{~dB}$ cuando se entra al espacio público). No obstante, esto se debe simplemente a la cercanía con el emisor 1. Cuando la fuente de emisión es el emisor 2, no se aprecian diferencias sensibles entre las instancias de ingreso al espacio público.

\section{Acceso indirecto 2}

Es un acceso en rampa que se encuentra ubicado en la parte media del EP1 con orientación noreste-suroeste. La visibilidad desde la instancia de aproximación es de $78 \%$, del $93 \%$ para la trascendencia y del $99 \%$ para la permanencia, permitiendo ver gran parte de la superficie de la plaza previamente a acceder a ella. En cuanto a la dispersión sonora, las instancias de aproximación registraron 53,2 dB (emisor 1) y 55,4 $\mathrm{dB}$ (emisor 2). Las instancias de trascendencia registraron valores de $55,4 \mathrm{~dB}$ (emisor 1) y $54,5 \mathrm{~dB}$ (emisor 2). Para las instancias de permanencia los valores registrados son de $57 \mathrm{~dB}$ (emisor 1) y $60 \mathrm{~dB}$ (emisor 2). Esto es, las voces generadas en el EP1 son audibles desde las tres instancias indistintamente de la fuente de emisión.

En este acceso, la variación en la cantidad de decibeles entre las instancias de aproximación y permanencia son apenas perceptibles sea cual fuera el emisor considerado.

\section{Acceso directo 1}

Se encuentra ubicado sobre el lado sur del EP1. La superficie visible para la instancia de aproximación al EP1 (4\%) es marcadamente baja en comparación con las instancias de trascendencia (91\%) y permanencia (94\%). El modelo de dispersión que tiene como origen del sonido al emisor 1 muestra un valor de 51,7 dB para la instancia de aproximación, $60,1 \mathrm{~dB}$ para la trascendencia y $70 \mathrm{~dB}$ para la permanencia. Cuando el sonido es emitido desde el emisor 2, la aproximación registró valores de $43,7 \mathrm{~dB}$, la trascendencia 40,3 dB y la permanencia $46,4 \mathrm{~dB}$. Las voces generadas en el EP1 son audibles desde las tres instancias, más allá de la fuente de emisión.

La diferencia entre estar dentro del pequeño recinto al Sur y estar en el espacio público para las instancias de aproximación y permanencia son destacables. Esto se debe a un notable incremento en la superficie visible del espacio público, desde el punto de vista visual. Al tiempo que desde el punto de vista auditivo, se registra un notable incremento en el nivel de presión sonora registrado cuando tenemos en cuenta el emisor 1. Cuando el emisor es el número 2, el aumento en el nivel de presión sonora es no perceptible. 


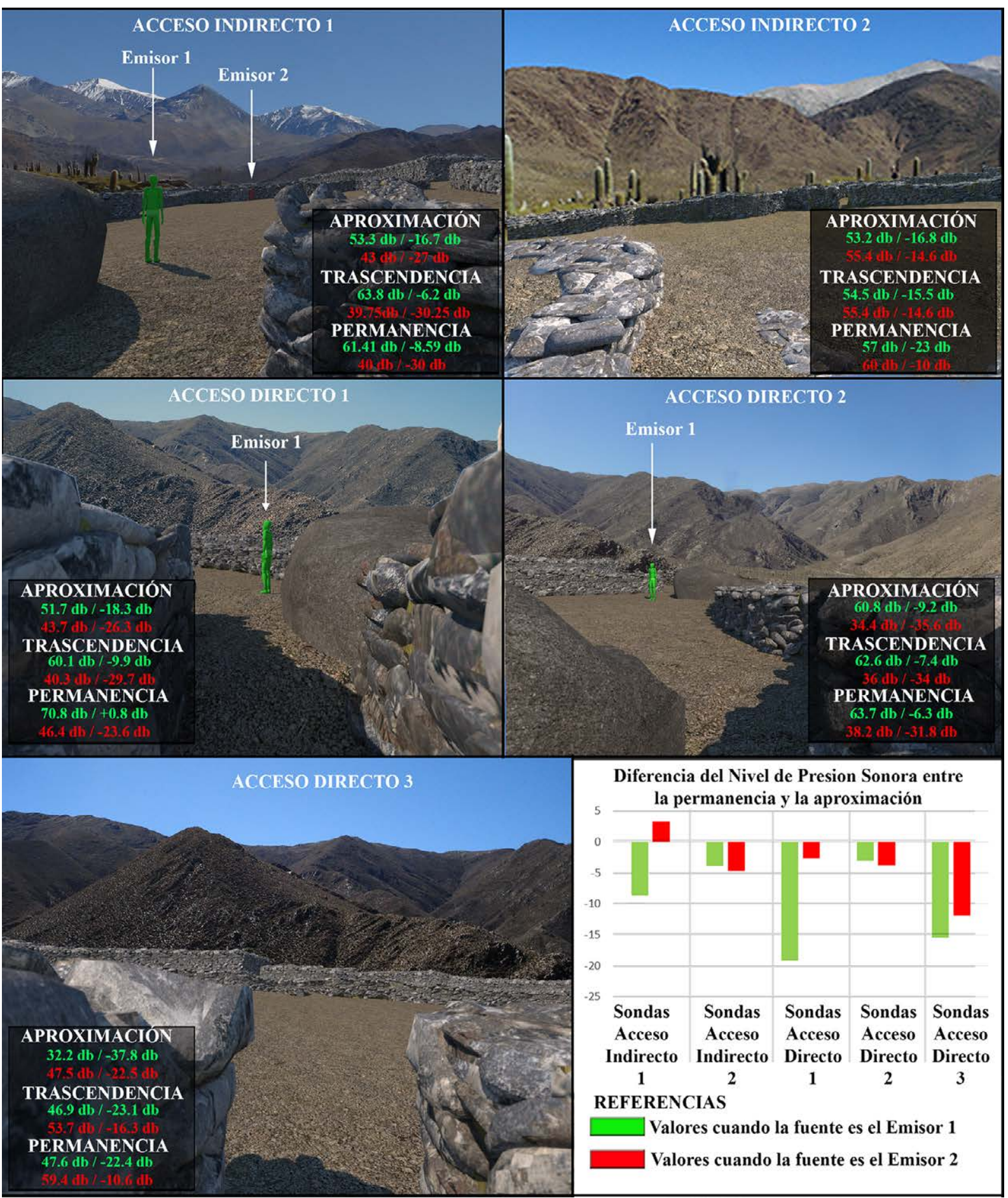

Figura 6. Valores del nivel de presión sonora de cada uno de los accesos y renderizado desde la instancia de aproximación. El gráfico representa la diferencia de decibeles entre la instancia de aproximación y permanencia de las sondas de cada acceso.

\section{Acceso directo 2}

Se encuentra ubicado hacia el suroeste del EP1. La visibilidad en las instancias de aproximación y trascendencia son bajas (20\% y $21 \%$ respectivamente), siendo mucho mayor para la permanencia (81\%). Para el modelo de dispersión sonora, cuando la fuente del sonido es el emisor 1, la instancia de aproximación registra $60,77 \mathrm{~dB}$, la instancia de trascendencia $62,63 \mathrm{~dB}$ y la instancia de permanencia $63,73 \mathrm{~dB}$. Cuando el 
sonido es emitido desde el emisor 2, el registro de la cantidad de decibeles de la instancia de aproximación es de 34,43 dB, el de la instancia de trascendencia es de 35,98 dB y el de la instancia de permanencia es de 38,23 dB. En definitiva, las voces generadas en el EP1 son audibles desde las tres instancias se cual fuese la fuente de emisión. A su vez, las instancias de aproximación y permanencia muestran un incremento apenas perceptible (emisor 2) o directamente imperceptible (emisor 1).

\section{Acceso directo 3}

Se encuentra ubicado en la parte media del EP1 hacia el Suroeste. El análisis de visibilidad indica que desde la instancia de aproximación solo sería visible un $24 \%$ del EP1, aumentando considerablemente en la trascendencia con $79 \%$ y en la permanencia con $84 \%$. El modelo de dispersión sonora mostró lecturas de 33,2 dB (emisor 1) y de 47,5 dB (emisor 2) en la instancia de aproximación. En la instancia de trascendencia se registraron $46,9 \mathrm{~dB}$ (emisor 1) y $53,7 \mathrm{~dB}$ (emisor 2), mientras que en la instancia de permanencia los valores fueron de $47,6 \mathrm{~dB}$ (emisor 1) y 59,4 dB (emisor 2). Al igual que en todos los casos aquí considerados, las voces generadas por ambos emisores en el EP1 son audibles en las tres instancias.

Deseamos destacar que este es el único acceso que no sólo muestra un incremento notable en la superficie visible del EP1 entre la instancia de aproximación y permanencia, sino que además registra una diferencia notable en los decibeles registrados independientemente de qué emisor se trate. En este acceso, con ambos emisores, hay un incremento en la cantidad de decibeles registrados entre la instancia de aproximación y permanencia.

\section{Estructuras alrededor del EP1}

Otro aspecto que evaluamos es el área de influencia del EP1 (Figura 7). Para ello, testeamos desde qué estructuras podría haber sido audible lo que sucediera en este espacio. Para realizar los análisis completamos el modelo tridimensional con 36 sondas ubicadas en el centro de cada una de las estructuras aledañas al EP1 (Figura 7). Recordamos que el EP1 se encuentra altitudinalmente por debajo de las estructuras al Norte y al Oeste y que configuramos la altura de los muros en 1,5 m. Esto implica que la visión desde los recintos aledaños hacia el EP1 podría haber quedado inhibida por defecto. Para estos modelos de dispersión sonora no interesa tanto la disparidad de lecturas dependiendo del emisor sino evaluar si las voces emitidas serían capaces de alcanzar los diferentes recintos y el nivel de presión sonora registrado en cada caso.

Los receptores del modelo de dispersión sonora ubicados en las estructuras al Suroeste (sondas 1 a 7) registran en promedio valores de $40 \mathrm{~dB}$ desde el emisor 1 y $38 \mathrm{~dB}$ desde el emisor 2. Ninguna de las sondas de este sector dio lecturas por debajo del índice de audición. Las sondas ubicadas al Noroeste, Oeste y Suroeste (sondas 8 a 22) registraron en promedio entre $37 \mathrm{~dB}$ (emisor 1) y $32 \mathrm{~dB}$ (emisor 2). Varias de las sondas de este sector no lograron registrar lecturas cuando el sonido se emite desde emisor 2. Las nueve sondas ubicadas en los recintos al Noroeste, Norte y Noreste del EP1 que lograron registrar sonidos audibles (sondas 23, 24, 28 y 30 a 35) poseen promedios de $32 \mathrm{~dB}$ (emisor 1) y $30 \mathrm{~dB}$ (emisor 2).

Al evaluar el alcance del EP1 se pudo determinar que el sonido tiene un amplio alcance. De las 36 sondas que circundan al EP1, solo cuatro de ellas no lograron registrar valor alguno (Sondas $25,27,29$ y 36 ) y el valor de la sonda 26 está por debajo del umbral de audición. Si bien las actividades realizadas no serían visibles desde el centro de los recintos aledaños, las voces emitidas en el EP1 podrían haber sido escuchadas desde la gran mayoría de los recintos aledaños. 


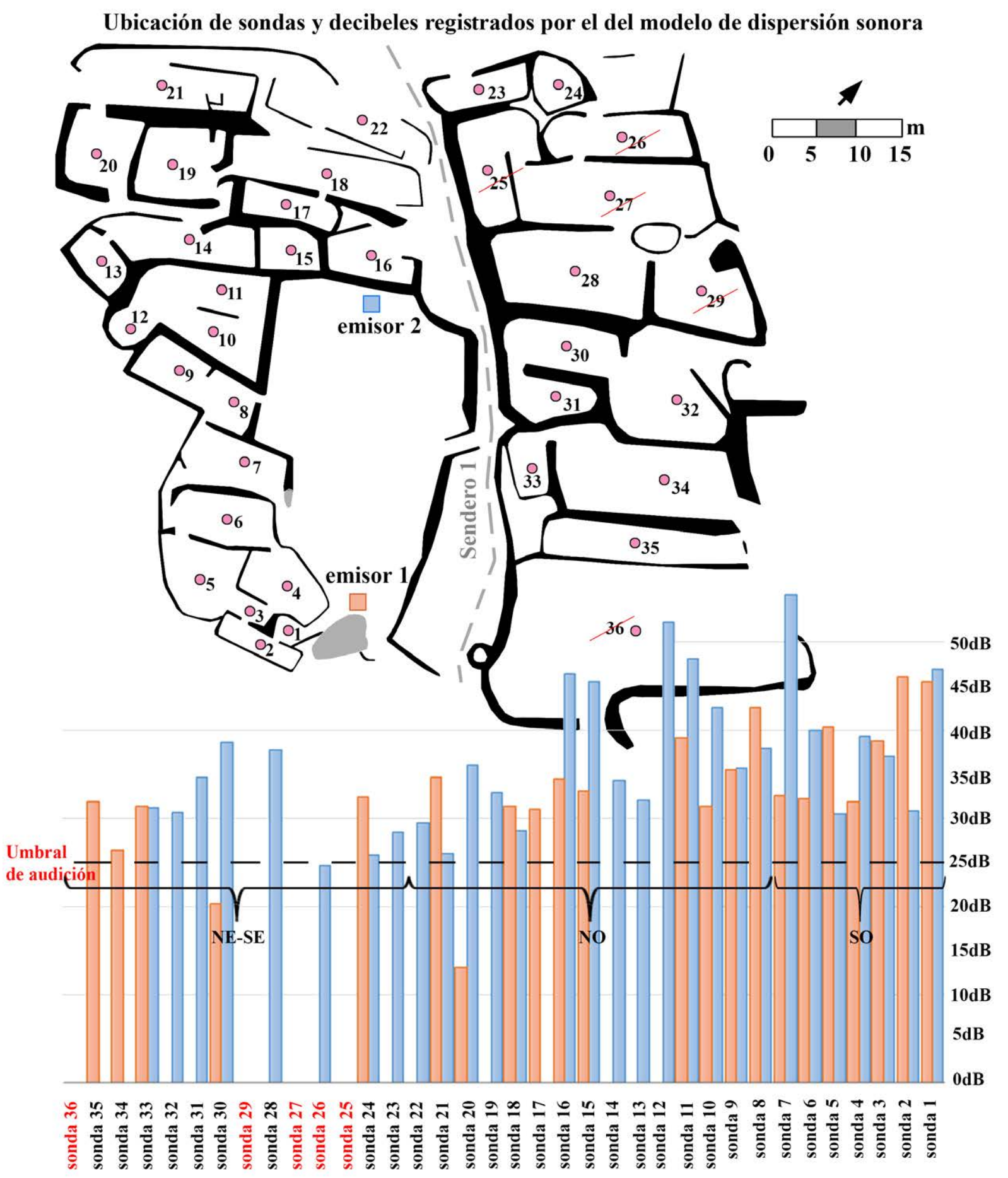

Figura 7. Ubicación de las sondas y gráfico que representa los decibeles registrados por cada una de ellas. En rojo y tachadas se resaltan las sondas cuyas lecturas son nulas o caen por debajo del umbral de audición.

\section{Escenario 2: Análisis acústico y visual del sendero 1}

El sendero 1 posee una longitud total de $104 \mathrm{~m}$. En este estudio sólo se consideran los $60 \mathrm{~m}$ que atraviesan nuestro recorte del sector. Nuestro interés aquí radica en entender cuánto se podría haber visto y escuchado de los recintos aledaños cuando se recorría este tramo del camino. 
El análisis de visibilidad acumulada (Figura $8 \mathrm{~A}$ ) indica que al transitar el sendero los recintos con los emisores 3 y 5 y el EP1 que se encuentran hacia el suroeste son vistos casi en su totalidad. Por otra parte, ninguno de los recintos al noreste, este y sureste del Sendero 1 sería visible desde el camino. Al modelo de dispersión sonora se agregaron 11 emisores que se encuentran ubicados en cada una de las estructuras que limitan con él: cinco de ellas se encuentran ubicadas en recintos al noroeste y suroeste del camino (emisores 1 al 5), mientras que ocho se ubican en las estructuras al noreste, este y sureste (emisores 6 al 13). Emplazamos nueve sondas que registran en simultaneo las lecturas de los 13 emisores (Tabla 2 y Figura 8B, C y D).

Las nueve sondas que se encuentran en el sendero (Figura 8B) lograron registrar el sonido de todos los emisores que se encontraban al Noroeste y Suroeste (emisores 1 al 5) y solo de algunos provenientes de los emisores ubicados al Noreste, Este y Sureste del camino (emisores 6 al 13, frecuentemente con valores por debajo del límite de audición, negrita en Tabla 2). No obstante, como se aprecia en la Tabla 2, todos los emisores al Noreste, Este y Sureste del camino lograron ser registrados con valores audibles por al menos una de las nueve sondas del camino. La sonda 1, ubicada en la parte más elevada del sendero, es la que recibe mayor cantidad de lecturas, recibiendo valores de cinco de los ocho emisores ubicados en las estructuras al Noreste, Este y Sureste del camino. Desde este punto del sendero se escucha el sonido proveniente de la mayor cantidad de estructuras. Le siguen las sondas 6,7 y 8 , ubicadas en la parte media/baja del sendero (Figura 8D).

En conjunto, estos resultados nos llevan a proponer que un sujeto que recorriera el sendero lograría ver lo que ocurre en el EP1 y en los recintos con emisores 3 y 5 (noroeste del camino), mientras que la visión hacia el Noreste, Este y Sureste estaría totalmente obstruida (recintos con emisores del 6 al 13). En otros términos, parece haberse privilegiado la accesibilidad visual al EP1 y no a los recintos aledaños. Respecto al modelo de dispersión sonora, las voces que se generan en las estructuras que se encuentran al Noroeste y Suroeste (emisores 1 a 5 en Figura 8A) son plenamente audibles desde el camino a lo largo de todo su recorrido. Por otro lado, a medida que uno recorre el sendero escucha las voces provenientes de algunas estructuras del Sureste, Este y Noreste, para luego dejar de escucharlas y escuchar el sonido proveniente de otras estructuras que antes no eran audibles. De este lado del camino, se destacan los recintos con los emisores $8,11, y 13$, que son los que mayor alcance tienen, con valores audibles registrados por siete de las nueve sondas en el sendero (Figura 8C). Es decir, las voces emitidas desde estas estructuras se escuchan en diversas partes del recorrido.

\section{Consideraciones finales}

Los escenarios testeados buscaron poner a prueba diferentes formas en que la simulación multifísica permite evaluar la dispersión del sonido y la proyección de la vista en escenarios controlados. Los resultados presentados aquí, aunque susceptibles de ser ajustados con mayor información arquitectónica (en particular la presencia o ausencia de techumbre), la determinación definitiva del comportamiento de ciertas variables y excavaciones extensivas que permitan determinar áreas de actividad y posicionar hipotéticamente a las personas, ilustran que la accesibilidad visual puede no ser suficiente para determinar la permeabilidad general de las estructuras.

Esto es particularmente relevante en los casos planteados. En el Escenario 1, pudimos ver que los dos emisores ubicados en los extremos de la plaza son plenamente visibles y audibles sin importar la fuente de emisión. Pudimos, además, identificar diferentes tendencias al analizar los accesos. En primer lugar, con solo acercarse a los accesos indirectos, la visibilidad del EP1 sería alta a muy alta ( $78 \%$ al 91,4\%). Por el contrario, 


\begin{tabular}{|l|c|c|c|c|c|c|c|c|c|c|c|c|c|}
\cline { 2 - 12 } \multicolumn{1}{c|}{} & \multicolumn{4}{|c|}{ Al Oeste del camino } & \multicolumn{6}{c|}{ Al Este del camino } \\
\hline Sonda/emisor & $\mathbf{1}$ & $\mathbf{2}$ & $\mathbf{3}$ & $\mathbf{4}$ & $\mathbf{5}$ & $\mathbf{6}$ & $\mathbf{7}$ & $\mathbf{8}$ & $\mathbf{9}$ & $\mathbf{1 0}$ & $\mathbf{1 1}$ & $\mathbf{1 2}$ & $\mathbf{1 3}$ \\
\cline { 2 - 12 } Sonda 1 & 27,1 & 32,4 & 32,6 & 33,9 & 52,7 & 38 & 31,8 & 38,1 & 32,2 & $\mathbf{2 1 , 4}$ & na & na & na \\
Sonda 2 & 36,9 & 32 & 32,2 & 37,2 & 63,7 & na & na & na & na & na & $\mathbf{1 4 , 8}$ & na & $\mathbf{2 2 , 3}$ \\
Sonda 3 & 38,7 & 36,8 & 64,5 & 56,6 & 55,3 & na & na & na & na & na & 30,4 & $\mathbf{2 3 , 5}$ & na \\
Sonda 4 & 43,3 & 42,5 & 59,9 & 36 & 41,9 & na & na & na & na & na & 25,9 & na & 27 \\
Sonda 5 & 37,4 & 47,6 & 37 & 34 & 44 & na & na & na & na & na & na & na & $\mathbf{1 1}$ \\
Sonda 6 & 42,1 & 53,9 & 35,6 & 39,7 & 40,1 & 31,3 & na & 32,5 & na & na & 55,4 & 26,2 & na \\
Sonda 7 & 57,2 & 39,7 & $\mathbf{1 2 , 9}$ & 38,2 & 38,3 & $\mathbf{2 3 , 7}$ & na & 32,6 & na & na & 43,2 & na & 36,7 \\
Sonda 8 & 35,5 & 45,4 & 31,3 & 32,4 & 37,3 & na & na & 26,3 & na & $\mathbf{2 4 , 1}$ & 35,6 & 34,3 & na \\
Sonda 9 & 32,4 & 43,8 & $\mathbf{2 9 , 3}$ & 28,7 & $\mathbf{2 7 , 8}$ & na & na & na & na & 32 & $\mathbf{2 2 , 7}$ & na & 44,4 \\
\hline
\end{tabular}

Tabla 2. Valores registrados (en dB) por las sondas del escenario 2. Se destacan los valores que caen por debajo del rango de audición.

cuando uno se aproxima a los accesos directos, la visibilidad del EP1 sería reducida a muy reducida ( $7 \%$ al $24 \%)$, incrementándose progresivamente a medida que se entra en él. Esto nos indica que podría haber existido cierta intención de inhibir la intervisibilidad entre el EP1 y el interior de aquellos recintos cuyos accesos directos los conectan con el espacio público. Pese a estas disparidades, las fuentes emisoras aquí testeadas habrían sido audibles en cualquiera de las instancias de cada acceso.

Por supuesto, estar en el interior del espacio público hubiese implicado, en la mayoría de los casos, percibir más decibeles que estando tras sus accesos. En un único caso (Acceso Indirecto 1) cuando el sonido es emitido desde el emisor 2, los decibeles registrados disminuyen en la instancia de permanencia, aunque esta disminución es imperceptible.

Por otra parte, dado que situamos ambos emisores en los extremos, quizás el aspecto más interesante surge de considerar qué sucedía con aquellos accesos que se ubican hacia la sección media del EP1, sobre el lado noreste (acceso indirecto 2) y sobre su lado suroeste (accesos directos 2 y 3 ) en relación a las dos fuentes emisoras propuestas. Como habíamos señalado, el acceso indirecto 2 se articula con una vía de circulación elevada respecto de EP1. Como es esperable, aproximarse a este acceso por el sendero debió haber permitido ver gran parte de la superficie de EP1 y sin presentar grandes variaciones en los $\mathrm{dB}$ percibidos entre las instancias de aproximación y permanencia. Los accesos directos 2 y 3 , por el contrario, poseían una visibilidad y audibilidad reducida en la instancia de aproximación y una diferencia notable y perceptible respecto del interior del EP1.

Con respecto del escenario 2, la proyección de la vista desde distintos puntos del sendero 1 expone otro aspecto interesante a considerar. Si bien la superficie interna de los recintos al noreste, este y sureste del sendero no es visible desde el camino, todos los emisores alcanzaron al menos una de las sondas del camino con valores superiores al umbral de audición. Las voces generadas en estos recintos son audibles desde gran parte del trayecto del sendero, especialmente en la parte alta (ubicación sonda 1) y media del camino (sondas 6,7 y 8 ).

Avanzar hacia la integración de la dispersión sonora en los asentamientos arqueológicos no está exento de limitantes. Uno de los aspectos más importantes es, sin duda, determinar definitivamente la altura de los muros de las estructuras y la existencia o no de techumbre (y sus materiales). Desconocemos además la clase de sonidos que allí se 


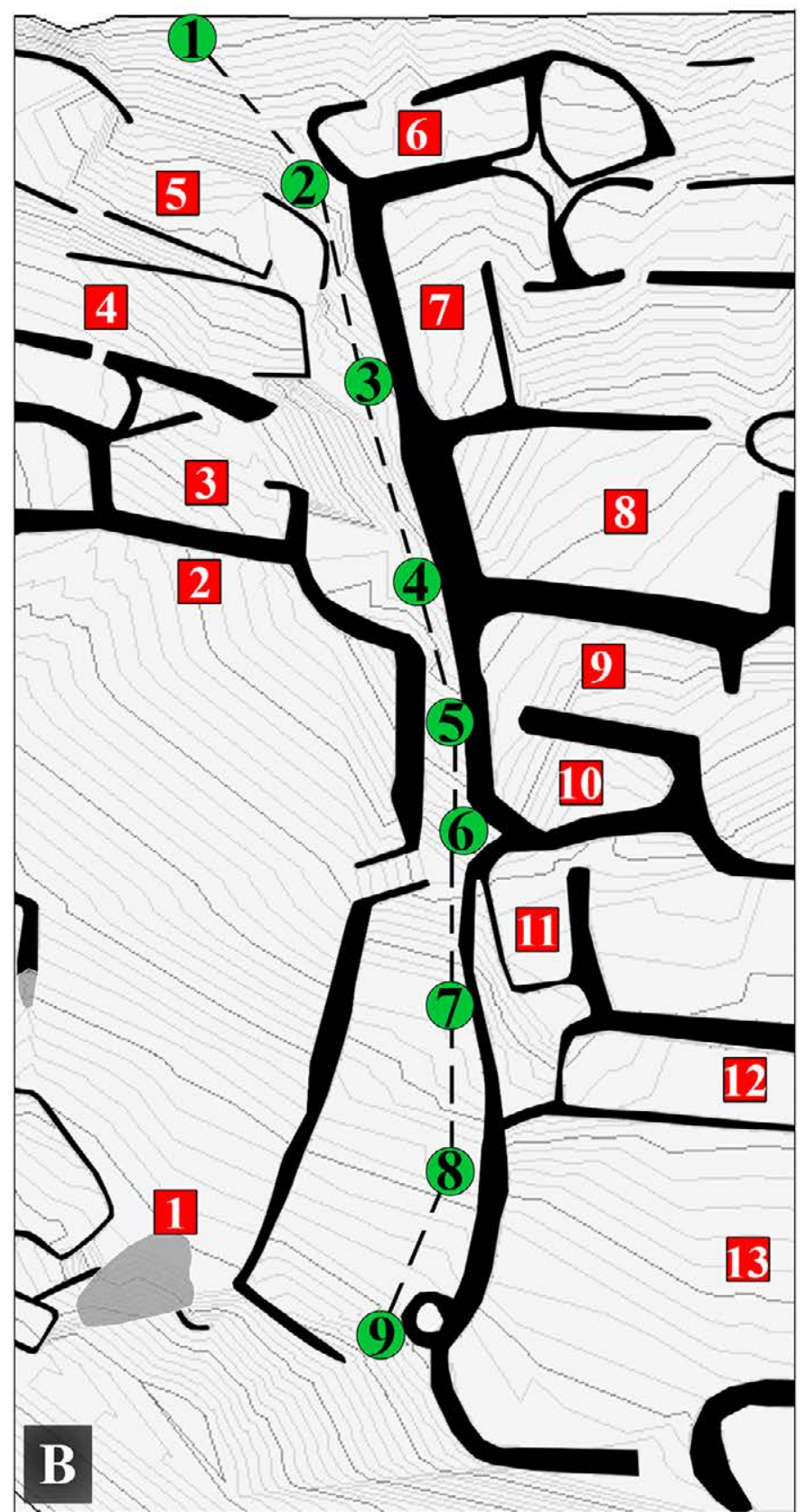

EMISORES

SONDAS

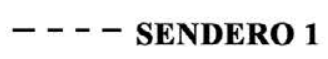

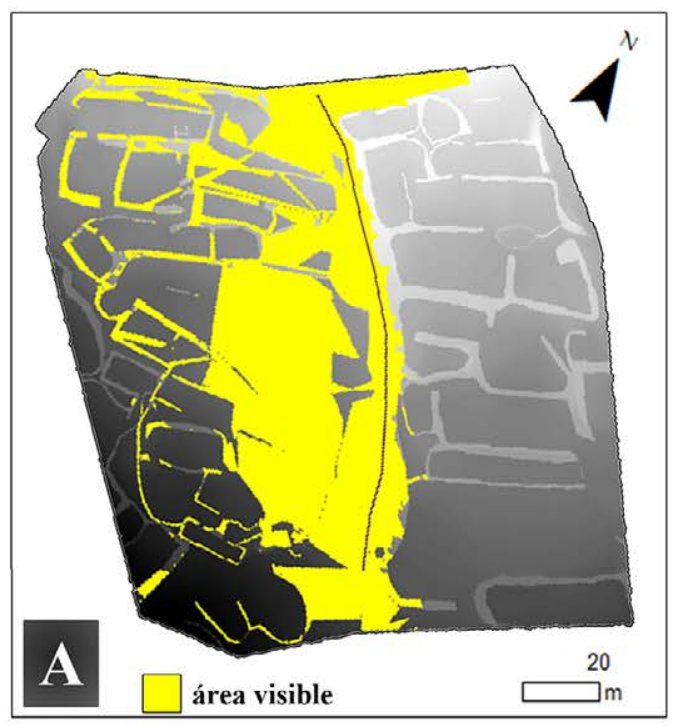
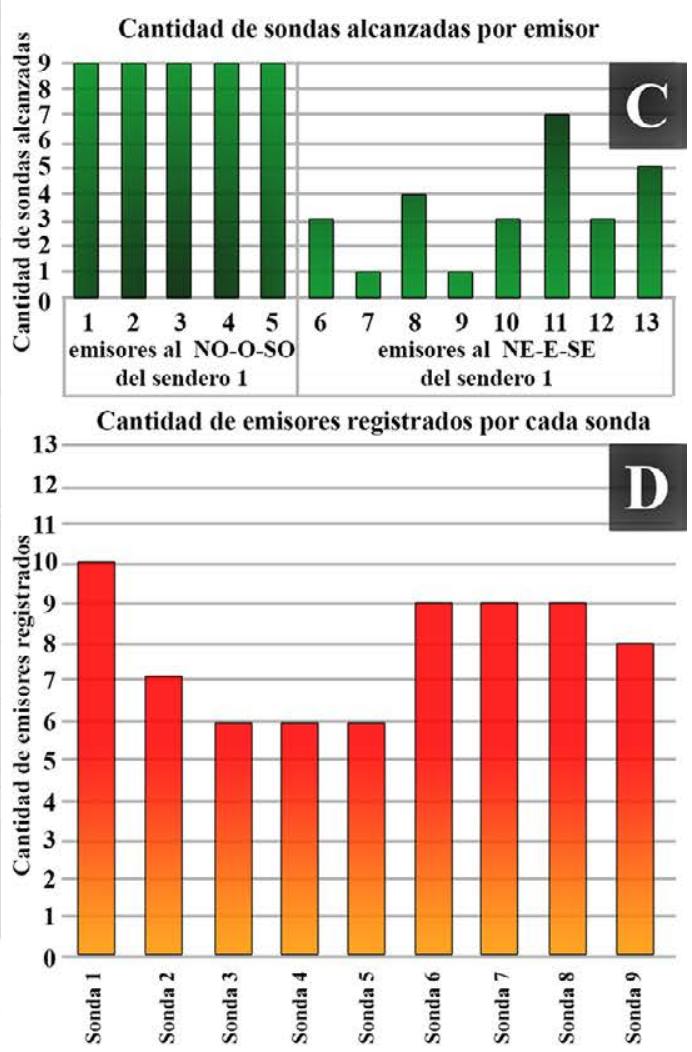

Figura 8. A) Distribución de sondas y emisores del escenario 2; B) Visibilidad acumulada desde el sendero; C) Sondas alcanzadas por cada emisor; D) Cantidad de emisores registrados por cada sonda.

emitían, si se utilizaban o no instrumentos, cantos, y si acaso existía una configuración específica para la distribución de personas que pudiera crear tal cosa como una división tajante entre orador/es y una audiencia. Otro aspecto, nada menor, es la incertidumbre respecto del movimiento de las personas y la cantidad de sujetos que pudieron estar presentes en el espacio público, considerando que más sujetos actuarían multiplicando la cantidad de emisores a la vez que atenuarían y modificarían la trayectoria e intensidad 
de los vectores acústicos. En este trabajo simplemente hemos buscado exponer el potencial de la simulación de la dispersión sonora y su importancia a la hora evaluar las propiedades de los ambientes construidos. Al integrarlos a otros análisis tradicionales de visibilidad buscamos ejemplificar el potencial de estos enfoques y marcar algunas limitaciones en las que debemos trabajar para avanzar en esta dirección. Futuros trabajos, con problemáticas más orientadas al caso específico y menos a las técnicas empleadas, continuarán generando datos para avanzar en la discusión respecto del rol de la arquitectura en la vida cotidiana de Las Pailas.

\section{Agradecimientos}

Este trabajo fue posible gracias al financiamiento de Wenner Gren Foundation for Anthropological Research, el Consejo Nacional de Investigaciones Científicas y Técnicas (CONICET - Argentina), y la Universidad Austral. Además, contó con el apoyo logístico de la comunidad Diaguita Kallchakí de Las Pailas. Finalmente, queremos agradecer a los evaluadores quienes ayudaron a mejorar este artículo con sus comentarios y sugerencias. 


\section{Referencias citadas}

» Benedikt, M. L. (1979). To take hold of space: isovists and isovist fields. Environment and Planning $B, 6,47-65$.

»Cátedra de acondicionamiento acústico. (2010). Tabla de absorción acústica de diversos materiales. Carrera de Arquitectura, Universidad de la República de Uruguay . http:// www.fadu.edu.uy/acondicionamiento-acustico/wp-content/blogs.dir/27/files/2012/o2/ Tablas-de-Absorcion.pdf (Acceso: 27 de Junio de 2010) Classen, C. (1997). Foundations for an anthropology of the senses. International Social Science Journal, 153(49), 401-412.

»Cripps, P. (2007). Pathways, Perception and the development of place. Computational approaches to movement and perception of landscapes in prehistory. Proceedings of XXXIII Computer applications in archaeology (pp. 323-326). Portugal: Tomar.

» Feld, S. (1996). Waterfalls of Song: An Acoustemology of Place Resounding in Bosavi, Papua New Guinea. En S. Feld y K. Basso (Eds.), Senses of Place (pp. 91-135). Santa Fe: School of American Research Press.

» Ferrari, A., Leibowicz, I., Izaguirre, J. y Acuto, F. (2017). Arquitectura y paisaje sonoro de un asentamiento Inka en el Noroeste Argentino. Chungara, Revista de Antropología, Chilena, 49, (3), 309-325.

"Forinash, K. (2015). Sound, An interactive ebook on the physics of sound. https:// soundphysics.ius.edu/ (Acceso: 29 de diciembre, 2017).

» Hall, E. T. (1959). The silent language. Nueva York: Doubleday \& Company.

» Hamilakis, Y. (2011). Archaeologies of the Senses. En T. Insoll (Ed.), The oxford Handbook of the Archaeology of Ritual y Religion (pp. 208-225). Oxford: Oxford University Press.

» Kergaravat, M., Ferrari, A. y Acuto, F. (2015). Dinámica social y estructuración del espacio en el valle Calchaquí Norte (Salta), durante el período tardío. Arqueología 21(1), 89-109.

» Kolar, A. M., Rick, J. W., Cook, P. R. y Abel, J. S. (2012). Ancient Pututus Contextualized: Integrative Archaeoacustics at Chavín de Huantar, Peru. En M Stöckli y A. A. Both (Eds.), Flower World Vol 1, Music Archaeology of the Americas (Volumen I) (pp. 23-53). Berlín: Ekho verlag.

"Nyberg, J. (2010). A peaceful sleep and Heavenly celebration for the pure and innocent. The sensory experience of death during the long Eighteenth Century. En F. Fahlander y A. Kjellström (Eds.), Making Sense of Things, Archaeologies of Sensory Perception (pp. 1533). Estocolmo: Edita Västra Aros AB.

»Parrondo Gayo, J. L., Velarde Suárez, S., González Pérez, J., Ballesteros Tajadura, R. y Santolaria Morros, C. (2006). Acústica medioambiental. Oviedo: Ediuno.

»Sanders, D. (1990). Behavioral conventions and archaeology: methods for the analysis of ancient architecture. En S. Kent (Ed.), Domestic architecture and the use of space. An Interdisciplinary cross-cultural study (pp. 43-72). Cambridge: Cambridge University Press.

» Scharf, B. (1978). Loudness. En E. C. Carterette y M. P. Friedman (Eds.), Handbook of Perception, (Volumen 4) (pp. 187-234). Nueva York: Academic Press.

»Schafer, R. M. (1969). The New Soundscape. A Handbook for the Modern Music Teacher. Ontario: Berandol Music Limited. 
"Scullin, D. yBoyd, B. (2014). Whistles in the wind: the noisy Moche city. World Archaeology, 46(3), 362-379.

"Seeger, A. (1975). The meaning of body ornaments. Ethnology, 14(3), 211-224.

" Takeshima, H., Susuki, Y., Ozawa, K., Kumagai, M y Sone, T. (2003). Comparison of loudness functions suitable for drawing equal-loudness-level contours. Acoustic Science and Technology, 24(2), 62-68.

» Tarragó, M. N. y Nuñez Regueiro, V. A. (1972). Un diseño de investigación arqueológica sobre el valle Calchaqui: fase exploratoria. Estudios de Arqueología, 1, 62-85.

" Tarragó, M, N. (1977). La localidad arqueológica de Las pailas, Provincia de Salta, Argentina. Actas del VII Congreso de Arqueología de Chile. Altos de Vilches, (Tomo 2), (pp. 499-517). Valdivia: Ediciones Kultrún.

"Van Ede, Y. (2009). Sensuous Anthropology: Sense and sensibility and the rehabilitation of Skill. Journal of Anthropological Notebooks, 15(2), 61-75. 